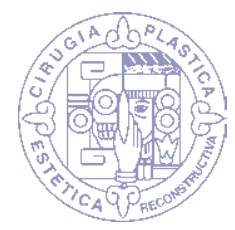

\title{
Congestión venosa de colgajo: acceso y tratamiento con énfasis en masaje como método costo-efectivo
}

\author{
Venous flap congestion: approach and treatment with \\ massage emphasis as a cost-effective method \\ Dr. Enrique Alonso Chaparro-Aranguren, * Dr. Alexander Leguía, \\ Dr. Sergio Alberto Malagón-Gaitán ${ }^{\S}$
}

Palabras clave: Congestión venosa, sufrimiento del colgajo, masaje, neoplasias de la mama, mamoplastia.

Keywords: Venous congestion, flap suffering, massage, breast neoplasms, mammaplasty.

* Cirujano plástico, equipo de cirugía plástica, Instituto Nacional de Cancerología, Bogotá, D.C., Colombia. Hospital Departamental de Villavicencio,

Villavicencio, Colombia, Centro de Investigaciones Oncológicas, Clínica San Diego, Bogotá, D.C., Colombia.

‡ Médico cirujano, Maestro en Farmacología y residente en cirugía plástica. Facultad de Medicina, Universidad Nacional de Colombia, Bogotá, D.C., Colombia. § Médico cirujano, Facultad de Medicina, Universidad Nacional de Colombia, Bogotá, D.C., Colombia.

Recibido: 02 agosto 2021 Aceptado: 18 agosto 2021

\section{RESUMEN}

La congestión venosa es una complicación frecuente en los colgajos, ya que una obstrucción venosa propicia la acumulación de sangre dentro del tejido y un flujo de salida insuficiente. El diagnóstico temprano es muy importante para intervenir precozmente con técnicas quirúrgicas y no quirúrgicas, con la perspectiva de cirugía plástica reconstructiva oncológica, dando énfasis en el masaje y su mecanismo de acción fisiopatológico. Realizamos una búsqueda bibliográfica en PubMed, BIREME y Google Scholar, usando los términos congestión venosa, sufrimiento del colgajo, masaje, neoplasias mamarias y mamoplastia, en inglés y español. Se incluyeron artículos de revisión y de resultados de investigación de 1980 a 2021. Después de la revisión y análisis de la información, concluimos que existen diversos métodos para la evaluación de la congestión venosa que permiten un tratamiento precoz, destacando el masaje como una medida terapéutica costo-efectiva con soporte fisiopatológico, cuyo propósito es mejorar la congestión venosa, evitar el sufrimiento del colgajo y mejorar los resultados para el paciente.

\section{ABSTRACT}

Venous congestion is a frequent complication in flaps, since a venous obstruction favors the accumulation of blood within the tissue and an insufficient outflow. Early diagnosis is very important to intervene in advance with surgical and non-surgical techniques, with the perspective of oncological reconstructive plastic surgery, emphasizing on massage and its pathophysiological mechanism of action. We carried out a bibliographic search in PubMed, BIREME and Google Scholar, using the terms venous congestion, flap suffering, massage, breast neoplasms and mammoplasty, in English and Spanish. Review articles and research results from 1980 to 2021 were included. After reviewing and analyzing the information, we conclude that there are various methods for the evaluation of venous congestion that allow early treatment, highlighting massage as a cost-effective therapeutic measure with pathophysiological support, with the purpose of improving the venous congestion, avoiding flap suffering and improving patient outcomes.

\section{INTRODUCCIÓN}

$\mathrm{L}$ Organización Mundial de la Salud reveló - que la incidencia de cáncer ha aumentado y actualmente el cáncer de mama es el más diagnosticado. ${ }^{1}$ En Colombia la conformación y oferta de centros de manejo oncológico está fragmentada y asimétrica, lo que conlleva a la necesidad de conformar una atención integral organizada y es fundamental incorporar a la cirugía plástica oncológica, ${ }^{2}$ la cual viene siendo partícipe con mayor frecuencia a través de colgajo regional o mediante colgajo libre. Sin embargo, a pesar de su alta tasa de éxito, se estima que cerca de 5-10\% de los casos requieren una nueva exploración cuando la viabilidad del colgajo se ve amenazada. ${ }^{3}$

Citar como: Chaparro-Aranguren EA, Leguía A, Malagón-Gaitán SA. Congestión venosa de colgajo: acceso y tratamiento con énfasis en masaje como método costo-efectivo. Cir Plast. 2021; 31 (2): 77-82. https://dx.doi. org/10.35366/102750 
Una de las principales complicaciones en esta técnica es la necrosis del colgajo, esencialmente ligado a la congestión venosa, en la cual la obstrucción del flujo venoso provoca un compromiso del colgajo. ${ }^{4}$

\section{MATERIAL Y MÉTODO}

Se realizó una búsqueda bibliográfica en PubMed, BIREME y Google Scholar, usando los términos venous congestion, flap suffering, massage, breast neoplasms, mammaplasty, en inglés y español. Se incluyeron artículos de revisión y de resultados de investigación desde 1980 hasta 2021 en todos los idiomas.

\section{Congestión venosa}

La congestión venosa es una causa común de falla del colgajo, siendo una complicación frecuente y desafiante. Si bien la obstrucción venosa parcial se puede compensar fisiológicamente mediante la neovascularización durante un periodo de tres a 10 días, la obstrucción total por colapso de la luz o trombosis venosa conduce en tres horas a lesiones microcirculatorias graves, y en ocho a 12 horas a lesiones microcirculatorias irreversibles. ${ }^{4}$

Desde el punto de vista fisiopatológico, en un inicio se produce el «fenómeno de no reflujo», donde la congestión venosa conduce a la acumulación de sangre dentro del tejido debido a un flujo de salida insuficiente, lo que conlleva a trombosis microcirculatoria mediante atrapamiento de plaquetas y estasis. De esta forma genera una oclusión gradual de los capilares y arterias, conllevando en las etapas finales a isquemia y necrosis tisular. ${ }^{4}$ La congestión venosa se relaciona con factores desencadenantes como espasmo venoso, anatomía venosa intrínseca, trombosis venosa, edema local y torsión pedicular. ${ }^{5}$

\section{Factores de riesgo}

El tabaquismo aumenta el riesgo de necrosis de la piel mediante el efecto vasoconstrictor directo de la nicotina, la oxigenación reducida de la hemoglobina y el aumento de la agregación plaquetaria. Se ha evidenciado que dejar de fumar antes de la cirugía reduce las complica- ciones postoperatorias. ${ }^{6}$ La cirugía previa de conservación de la mama (escisión local amplia y radioterapia), puede aumentar la incidencia de necrosis, presumiblemente mediada en parte por los efectos de la radioterapia previa de la pared torácica. ${ }^{6}$

Existen factores de riesgo con significancia estadística predictiva de necrosis como mayor peso de mastectomía y mayor peso del colgajo autólogo (representan una superficie más grande del colgajo con mayor volumen de la mama o más tensión del colgajo postreconstrucción), índice de masa corporal aumentado y diabetes mellitus (por potencial formación de anomalías vasculares, viscosidad sanguínea aumentada y disfunción endotelial).6,7

Dentro de los factores quirúrgicos se incluye el tipo de incisión, disminución del grosor del colgajo, volumen alto de relleno del expansor tisular, duración extensa de la cirugía y la técnica en sí misma, esta última teniendo en consideración la importancia del conocimiento sobre la anatomía vascular. ${ }^{6}$

Los determinantes del grosor óptimo del colgajo dependen de obtener un equilibrio durante el procedimiento para lograr márgenes de resección claros, evitando que sean muy delgados. Esto se logra mediante una cuidadosa adherencia al plano oncoplástico entre la grasa subcutánea y el parénquima mamario. Una estrecha adherencia al mismo es crucial para lograr una resección sólida mientras se preserva el suministro de sangre a la piel..$^{8,9}$

Desafortunadamente, muchos factores de riesgo del paciente no son modificables entre el diagnóstico y la cirugía; sin embargo, en pacientes con alto riesgo de necrosis, la realización de una mastectomía simple con una reconstrucción retrasada con la administración oportuna de cualquier terapia adyuvante puede brindar más tiempo para modificar factores de riesgo ajustables antes de la reconstrucción.

\section{Evaluación del colgajo}

Dada la naturaleza irreversible de los cambios microcirculatorios en la insuficiencia venosa es primordial reconocerla de forma temprana, así el tejido puede tener mayor probabilidad de supervivencia debido a la relativa facilidad de identificación y al progreso lento de la misma. ${ }^{3}$ 
Esta evaluación se lleva a cabo principalmente mediante la evaluación clínica del suministro sanguíneo, del color de la piel, llenado capilar, temperatura y sangrado dérmico. ${ }^{10,11}$

No obstante, la evaluación clínica tiene sus limitaciones, lo que ha llevado a nuevas tecnologías que permiten identificar una perforante adecuada en el prequirúrgico, durante la cirugía o incluso en el postoperatorio para valorar la adecuada perfusión del tejido e identificar si hay sufrimiento. Entre ellas encontramos el Doppler portátil, ${ }^{12}$ flujometría Doppler láser, angiografía con fluoresceína, angiografía con verde de indocianina ${ }^{13}$ y nuevas técnicas que usan cámaras de termografía para valorar en tiempo real la perfusión del tejido. ${ }^{14,15}$

\section{Impacto}

El adecuado acceso influye en aspectos como el retraso en la terapia adyuvante, manejo de la herida, mal resultado estético y consecuencias emocionales para el paciente, en especial ansiedad y angustia resultantes. ${ }^{6}$ Añadido a ello, la carga financiera se ve aumentada, dada la necesidad de un mayor número de citas ambulatorias, implementos, equipos y la necesidad de reintervención quirúrgica, alcanzando hasta $50 \%$ de sobrecosto. ${ }^{16}$

\section{TRATAMIENTO}

La pronta identificación permite una intervención temprana, principalmente mediante la exploración quirúrgica, que en la actualidad es el estándar de oro. No obstante, en casos que no sea factible o falle, existen técnicas alternativas de rescate que propenden a aumentar la perfusión tisular y disminuir la congestión para evitar cambios persistentes en la microcirculación y que se produzca neovascularización entre el séptimo y décimo día postquirúrgico. ${ }^{5}$

Sanguijuelas medicinales. La especie más utilizada es Hirudo medicinalis, cuyas glándulas salivales contienen hirudina, un potente anticoagulante que inactiva la trombina al reemplazar el fibrinógeno y afecta el factor Xa que cataliza la conversión de protrombina en trombina. ${ }^{4}$ La actividad de la hirudina junto a otros inhibidores de la función plaquetaria hace posible el flujo de sangre mientras se produce la picadura, ${ }^{4}$ y el efecto mecánico de succión permite mejorar la perfusión al drenar activamente sangre del tejido. ${ }^{11}$

La frecuencia óptima de aplicación es de dos a ocho horas, mientras que la duración total promedio ha sido de cuatro a 10 días, con una tasa de éxito de 65 a 85\%; no obstante, 50\% de los casos requieren transfusión y es indicativo profilaxis antibiótica contra aeromonas. ${ }^{4} \mathrm{Se}$ debe tener en cuenta que su disponibilidad es limitada, lo que puede retrasar su aplicación hasta $24-48$ horas, ${ }^{4}$ su tasa de éxito disminuye considerablemente en colgajos grandes y no existen guías establecidas para su uso. ${ }^{11}$

Heparina subcutánea local. Se administra heparina de bajo peso molecular frecuentemente, se ajusta la dosis inicial dependiendo del área congestiva del colgajo, siguiendo un patrón decreciente, teniendo un esquema claro y reproducible. ${ }^{5,11}$ La terapia debe continuarse por un mínimo de 10 días; sin embargo, se relaciona con morbilidad asociada como el desarrollo de hematomas y necesidad de transfusiones, con un promedio de cinco unidades de glóbulos rojos por paciente. ${ }^{5}$

Terapia con presión negativa. Se ha aplicado presión negativa continua, promedio entre 100-150 mmHg, variando la presión dependiendo de la condición de los pacientes y la cantidad de flujo sanguíneo. La duración de la terapia oscila entre tres y 10 días. ${ }^{3}$ Se han descrito tres posibles mecanismos de acción: mejora del flujo sanguíneo local eliminando la congestión sanguínea intersticial; aumenta la tasa de revascularización entre los sitios donantes y receptores por medio de la neoangiogénesis; reduce la presión del espacio intersticial eliminando el exceso de líquido y coágulos del lecho de la herida. ${ }^{3}$ Se ha de tener en cuenta que su uso continuo genera compresión del pedículo y por ende, insuficiencia arterial del colgajo, sumado a un efecto compresivo, por ello se aconseja que se utilice en la modalidad de succión discontinua. ${ }^{11}$

\section{MASAJE MANUAL}

Impacto fisiopatológico del masaje. La terapia con masaje altera significativamente la bioquímica de los tejidos tras cada sesión terapéutica a través de distintos mecanismos. Influye en la actividad simpática a través del 
sistema hipotalámico-hipofisario-adrenal, aumentando los niveles de serotonina y dopamina, que a su vez disminuyen los niveles de cortisol, mismo que tiene un papel negativo en la función inmunológica, disminuye el número de células NK (Natural Killer) y linfocitos relevantes en la respuesta de defensa en cáncer, en particular en mama. ${ }^{17}$ Se ha encontrado un aumento significativo en la respuesta inmunológica Th1 que repercute significativamente en la relación Th1/Th2, siendo un índice importante de la función inmunológica que representa una menor producción de citoquinas. ${ }^{18}$

Otro efecto es la descomposición mecánica de la necrosis grasa en desarrollo, que la convierte en pequeños fragmentos, de esta manera mejora la eliminación del tejido necrótico por el sistema linfático y evita calcificaciones, lo que permite cambios en la mama haciendo que adapte una forma más suave, regular y lisa, evitando así complicaciones subsecuentes. ${ }^{18,19}$

El masaje también influye en el manejo del linfedema, que genera una reducción continua del edema al evaluar el masaje manual y el masaje neumático. ${ }^{20}$ Se debe tener en cuenta la necesidad de una movilización temprana de la extremidad, ya que evidencia una mejor modulación del dolor y un mayor grado de movilidad del hombro en el postquirúrgico. ${ }^{21}$ Sin embargo, al comparar el ejercicio físico solo y el ejercicio asociado a masaje, se demostró que el masaje se debe incluir en el manejo, dada la mejoría en la cicatriz, la aducción del hombro y el edema. ${ }^{22,23}$

Se evidenció que no aumenta el riesgo de contractura capsular cuando se controlan otros factores asociados ${ }^{24}$ y se considera un método seguro. ${ }^{25}$ Hay que tener en cuenta que el masaje ha mostrado múltiples beneficios al ser evaluado en diversas patologías, provocando reducción del dolor, depresión, ansiedad, tensión y fatiga. ${ }^{17,18}$

Frecuencia de aplicación. Diferentes estudios evalúan la realización de masaje con variabilidad en la frecuencia de la intervención. Algunos recomiendan sesiones de 10 minutos tres veces al día en el manejo inicial postoperatorio inmediato para el manejo de complicaciones ${ }^{18}$ y otros aconsejan el manejo preventivo de complicaciones de forma más tardía. ${ }^{22}$ Otros aplican menor frecuencia en el manejo de complicaciones tardías. ${ }^{23,25,26}$

«Masaje cardiaco» - experiencia personal y propuesta costo-efectiva. La presión manual ejercida periódicamente por un tiempo aproximado de 72 horas mejora la irrigación del colgajo con sufrimiento. Se debe iniciar una vez que se identifique la congestión venosa y puede utilizarse como primera medida de tratamiento no quirúrgico. Basado en el masaje cardiaco directo, se ejerce presión fuerte y constante con las yemas de los dedos o con toda la mano, según el área del colgajo, un promedio de 50 contracciones por minuto seguidas de una pausa. La duración depende del grado de cianosis en casos severos 24 horas del día; en cianosis moderada durante una hora y descansar la hora siguiente; en cianosis leve se disminuye o aumenta la frecuencia del masaje según la evolución. Se sugiere que se disponga de otra persona dispuesta a realizarlo por la fatiga que puede generar al paciente y registrar por imagen para hacer vigilancia clínica estricta.

En nuestra experiencia su utilidad destaca en la cianosis fija del colgajo, aquélla que no cambia con la posición del paciente y que se debe diferenciar de la cianosis posicional. Esta última está presente cuando el paciente está de pie y se da por el efecto de la tracción que ejerce el peso del colgajo sobre el pedículo vascular. Esta tracción disminuye cuando el paciente se coloca en posición de decúbito supino, permitiendo así mejorar el retorno venoso.

\section{CONCLUSIÓN}

Son diversas las técnicas usadas para el manejo de la cianosis de los colgajos, unas más comunes por su facilidad y estudios que los aprueban. La revisión quirúrgica del colgajo se considera el «estándar de oro»; sin embargo, no siempre se tiene esta opción disponible, por lo que el conocimiento de otras técnicas no quirúrgicas puede llevar a proteger el colgajo. No obstante, algunos de estos métodos no siempre están disponibles y los resultados pueden no ser satisfactorios o presentan complicaciones asociadas que dificultan el tratamiento y prolongan la estancia hospitalaria. 
El reconocimiento de la literatura actual nos muestra los beneficios del masaje directo en el estado inmunológico, manejo del dolor, resultado estético, disminución de reintervención, reducción de sobrecosto y en especial en la condición psicológica del paciente.

Se brinda aporte teórico al uso del masaje, pues éste modula la fisiopatología de la congestión venosa encontrada en los colgajos con sufrimiento. Esta intervención puede ser reproducible mediante la técnica de masaje directo, la cual es costo-efectiva, no trae consigo mayores riesgos para el paciente y no requiere de experticia avanzada para su realización. Por ende, puede convertirse en parte del arsenal del manejo no quirúrgico ligado a una adecuada evaluación previa para calcular factores de riesgo y signos prematuros de congestión venosa que permitan un seguimiento estricto e instauración temprana. Esto nos permite abrir la posibilidad a nuevos estudios para su evaluación objetiva en casos de congestión venosa de colgajos y la necesidad de un estándar que permita su aplicación y comparación.

\section{REFERENCIAS}

1. Sung $H$, Ferlay J, Siegel RL, Laversanne $M$, Soerjomataram I, Jemal A et al. Global cancer statistics 2020: GLOBOCAN estimates of incidence and mortality worldwide for 36 cancers in 185 countries. CA Cancer J Clin 2021; 71 (3): 209-249. doi: 10.3322/ caac. 21660.

2. Murcia E, Aguilera J, Wiesner C, Pardo C. Oncology services supply in Colombia. Colomb Med 2018; 8996. doi: 10.25100/cm.v49i1.3620,

3. Qiu SS, Hsu C-C, Hanna SA, Chen SH-Y, Cheong C-F, $\mathrm{Lin} \mathrm{C}-\mathrm{H}$ et al. Negative pressure wound therapy for the management of flaps with venous congestion. Microsurgery 2016; 36 (6): 467-473. doi: 10.1002/micr.30027

4. Herlin C, Bertheuil N, Bekara F, Boissiere F, Sinna R, Chaput B. Leech therapy in flap salvage: Systematic review and practical recommendations. Ann Chir Plast Esthet 2017; 62 (2): e1-13. doi: 10.1016/j. anplas.2016.06.004

5. Pérez M, Sancho J, Ferrer C, García O, Barret JP. Management of flap venous congestion: the role of heparin local subcutaneous injection. J Plast Reconstr Aesthet Surg 2014; 67 (1): 48-55. doi: 10.1016/j.bjps.2013.09.003.

6. Robertson SA, Jeevaratnam JA, Agrawal A, Cutress RI. Mastectomy skin flap necrosis: challenges and solutions. Breast Cancer (Dove Med Press) 2017; 9: 141-152. doi: 10.2147/BCTT.S81712.

7. McCarthy CM, Mehrara BJ, Riedel E, Davidge K, Hinson A, Disa JJ et al. Predicting complications following expander/implant breast reconstruction: an outcomes analysis based on preoperative clinical risk. Plast Reconstr Surg 2008; 121 (6): 1886-1892. doi: 10.1097/PRS.0b013e31817151c4.

8. Robertson SA, Rusby JE, Cutress RI. Determinants of optimal mastectomy skin flap thickness: Mastectomy skin flap thickness. Br J Surg 2014; 101 (8): 899-911. doi: 10.1002/bjs.9470.

9. Beer GM, Varga Z, Budi S, Seifert B, Meyer VE. Incidence of the superficial fascia and its relevance in skin-sparing mastectomy. Cancer 2002; 94 (6): 16191625. doi: 10.1002/cncr.10429.

10. Smith JM, Zeebregts CJ, Acosta R, Werker PMN. Advancements in free flap monitoring in the last decade: a critical review. Plast Reconstr Surg 2010; 125 (1): 177-185. doi: 10.1097/PRS.0b013e3181c49580.

11. Boissiere F, Gandolfi S, Riot S, Kerfant N, Jenzeri A, Hendriks $S$ et al. Flap venous congestion and salvage techniques: a systematic literature review. Plast Reconstr Surg Glob Open 2021; 9 (1): e3327. doi: 10.1097/GOX.0000000000003327

12. Sylvester-Frías $M$, Roco-Molina $H$, Meruane-Naranjo M. Utilidad del doppler manual en la planificación del colgajo DIEP. Cir Plast Iberolatinoam [Internet] 2016; 42 (4): 371-378. Disponible en: http://scielo. isciii.es/scielo.php?script $=$ sci_arttext\&pid $=$ S037678922016000400009\&lng=es

13. Otormin G, Novello G, Gambini J, Juri H. Evaluación de la perfusión de colgajos mediante Indocianina verde. Cir Plast Iberolatinoam 2018; 44 (3): 303-309. doi.org/10.4321/s037678922018000300011.

14. Larrea-Prieto J, Núñez-Orduña E, Bendito-Guilarte B, Gianella C, Iglesias-Aguilar C, Miñón-Santamaría C. Concordancia entre termografía con teléfono inteligente y angio-tac para detección de perforantes cutáneas en colgajos DIEP. Cir Plast Iberolatinoam [Internet] 2020; 46 (1): 53-56. Disponible en: http://scielo.isciii.es/scielo.php?script $=$ sci_ arttext\&pid $=$ S0376-78922020000100008\&lng=es. Epub 25-Mayo-2020. https://dx.doi.org/10.4321/ s0376-78922020000100008.

15. Hennessy O, Potter SM. Use of infrared thermography for the assessment of free flap perforators in autologous breast reconstruction: a systematic review. JPRAS Open 2020; 23: 60-70. doi: 10.1016/j. jpra.2019.11.006.

16. Yalanis GC, Nag S, Georgek JR, Cooney CM, Manahan MA, Rosson GD et al. Mastectomy weight and tissue expander volume predict necrosis and increased costs associated with breast reconstruction. Plast Reconstr Surg Glob Open 2015; 3 (7): e450. doi: 10.1097/ GOX.0000000000000408.

17. Field T, Hernandez-Reif M, Diego M, Schanberg $\mathrm{S}$, Kuhn C. Cortisol decreases and serotonin and dopamine increase following massage therapy. Int J Neurosci 2005; 115 (10): 1397-1413. doi: 10.1080/00207450590956459.

18. Field T. Massage therapy research review. Complement Ther Clin Pract 2016; 24: 19-31. doi: 10.1016/j. ctcp.2016.04.005

19. Amir A, Silfen R, Hauben DJ. Fat necrosis after a transverse rectus abdominis myocutaneous flap? How about massage? Plast Reconstr Surg 2000; 106 (5): 
1216-1217. doi: 10.1097/00006534-20001000000050.

20. Zanolla R, Monzeglio C, Balzarini A, Martino G. Evaluation of the results of three different methods of postmastectomy lymphedema treatment. J Surg Oncol 1984; 26 (3): 210-213. doi: 10.1002/jso.2930260317.

21. Le Vu B, Dumortier A, Guillaume MV, Mouriesse H, Barreau-Pouhaer L. Efficacité du massage et de la mobilisation du membre supérieur après traitement chirurgical du cancer du sein [Efficacy of massage and mobilization of the upper limb after surgical treatment of breast cancer]. Bull Cancer 1997; 84 (10): 957-961.

22. Zhang L, Fan A, Yan J et al. Combining manual lymph drainage with physical exercise after modified radical mastectomy effectively prevents upper limb lymphedema. Lymphat Res Biol 2016; 14 (2): 104-108. doi: 10.1089//rb.2015.0036.

23. Cho Y, Do J, Jung S, Kwon O, Jeon JY. Effects of a physical therapy program combined with manual lymphatic drainage on shoulder function, quality of life, lymphedema incidence, and pain in breast cancer patients with axillary web syndrome following axillary dissection. Support Care Cancer 2016; 24 (5): $2047-$ 2057. doi: 10.1007/s00520-015-3005-1.

24. Stevens WG, Nahabedian MY, Calobrace MB et al. Risk factor analysis for capsular contracture: a 5-year Sientra study analysis using round, smooth, and textured implants for breast augmentation. Plast Reconstr Surg 2013; 132 (5): 1115-1123. doi: 10.1097/01. prs.0000435317.76381.68.

25. Oliveira MMF, Gurgel MSC, Amorim BJ et al. Long term effects of manual lymphatic drainage and active exercises on physical morbidities, lymphoscintigraphy parameters and lymphedema formation in patients operated due to breast cancer: a clinical trial. PLoS One 2018; 13 (1): e0189176. doi: 10.1371/journal. pone.0189176.

26. Martín ML, Hernández MA, Avendaño C, Rodríguez F, Martínez H. Manual lymphatic drainage therapy in patients with breast cancer related lymphedema. BMC Cancer 2011; 11: 94. doi: 10.1186/1471-240711-94.

Conflicto de intereses: Los autores declaran no tener conflicto de intereses.

Correspondencia:

\section{Dr. Enrique Chaparro-Aranguren}

Calle 23 Núm. 66-39, Interior 4-1002,

Bogotá, Colombia.

E-mail: chaparro.aranguren@gmail.com, echaparro@cancer.gov.co 